\title{
Effects of 1,8-Cineole on the Activity of Cyclooxygenase and Cyclooxygenase 1 and Cyclooxygenase 2 Isoforms
}

Beer $\mathbf{A M}^{*}$, Zagorchev P, Draganova-Filipova $\mathbf{M}$ and Lukanov $\mathbf{J}$

Ruhr University Bochum, Im Vogelsang 5-11, 45527 Hattingen, Germany

\begin{abstract}
Purpose: The anti-inflammatory effects of 1,8-cineole (eucalyptol) in the treatment of acute bronchitis and treatment of chronic airway diseases like asthma as well as hay fever have been observed in clinical practice for some time. The anti-inflammatory effect has been proven in numerous studies.
\end{abstract}

Material and methods: The effect of 1,8-cineole on the activity of cyclooxygenase and its two isoforms (COX-1 and COX-2) were analysed and compared with the effects of indomethacin and celecoxib.

Results: The ratio between the concentrations required to achieve $50 \%$ inhibition of COX-1 and COX-2 isoform activity was found to be 73.5 .

Discussion: For indomethacin, the ratio is 9.71 and for celecoxib 79.5 . For indomethacin, it is about 10 times less than that of 1,8-cineole.

Conclusion: The present study provides useful data suggesting that 1,8 -cineole is a specific COX-2 blocker.

Keywords: 1,8-cineole; Inflammation; Indomethacin; Celecoxib; Cyclooxygenase; Eucalyptol

\section{Introduction}

1,8 -cineole is found in many essential oils. It is the main ingredient of both eucalyptus (70\%) and rosemary oils, where its concentration can exceed 45\% [1]. Freeze drying and steam distillation, yields 99\% pure eucalyptus oil, from which a pure form of 1,8-cineole can be obtained. In vitro studies have revealed secretomotor, expectorant, antispasmodic $[2,3]$, local hyperaemic and anti-inflammatory properties [4] for naturally isolated 1,8-cineole (synonym: eucalyptol). Due to its efficacy and excellent tolerability, 1,8-cineole has been successfully used for decades for topical treatment of rheumatic disorders, as well as for oral application to treat inflammatory diseases of the respiratory tract, sinusitis, acute and chronic bronchitis and as a concomitant therapy in COPD and steroid-dependent asthma [5,6]. The work of Juergens et al. [7] has shown that 1,8-cineole inhibits the activity of cyclooxygenase.

It is known that most of the frequently used cyclooxygenase blockers also have undesirable side effects [8]. Nonsteroidal anti-inflammatory drugs (NSAIDs) have analgesic, antipyretic, and anti-inflammatory effects. They are in worldwide use, primarily for pain management. However, taking NSAIDs is also associated with a significant number of side effects. The side effects of taking NSAIDs are mainly mucosal lesions, dyspepsia, gastrointestinal bleeding, ulcers, perforations, and stenosis.

The aim of this study is to determine the extent to which 1,8-cineole influences the activity of cyclooxygenase (COX), COX-1 and COX-2 enzyme systems. The results were compared with the effects of indomethacin, an analgesic in the NSAID group that acts as a nonselective inhibitor of cyclooxygenase [9] (COX-1 and COX-2), and with the effects of celecoxib. Celecoxib is a selective COX-2 inhibitor that is used in the treatment of degenerative joint disease, chronic polyarthritis, and ankylosing spondylitis. It relieves pain, inflammation, and fever.

\section{Materials and Methods}

\section{COX-1 and COX-2 activity assay}

Changes in the activity of COX-1 and COX-2 isoforms of cyclooxygenase were measured with Standard Kit (Cayman Chemical, USA). COX activity assay is a colorimetrical assay of the peroxidase component of cyclooxygenases. Assay is based on the monitoring of the appearance of oxidized N,N,N',N'-tetramethyl-p-phenylenediamine (TMPD). According to the manufacturer protocol in each well of the 96-well plate the appropriated amount of Assay Buffer, Heme, standards and samples were added. The colorimetric substrate was added. To initiate the reaction arachidonic acid was used. After short incubation at room temperature the absorption was measured on the plate reader (Sunrise, Tecan, Austria) on $590 \mathrm{~nm}$.

Changes in light absorption were measured with a photometer at $590 \mathrm{~nm}$ and converted to changes in activity with the following equation:

$$
X_{n}^{\operatorname{COX}-1(2)}=100-\left(A_{n}^{\operatorname{COX} 1(2)} \cdot 100\right) / A^{\operatorname{COX}-1(2)}{ }_{100}
$$

Where $A^{\operatorname{Cox} 1(2)}$ is the absorption at concentration ' $n$ ' of the test substance

$A_{100}{ }^{C O X-1(2)}$ is the absorption at $100 \%$ activity of the enzymes

$\mathrm{n}=$ concentration of the substances in $\mathrm{M}$

$A_{n}{ }^{C O X(2)}=$ Light absorption at a wavelength of $590 \mathrm{~nm}$

$A_{n}{ }^{\operatorname{COX}(2)}=$ Light absorption at a wavelength of $590 \mathrm{~nm}$

$A_{100}{ }^{C O X(2)}=$ Light absorption at maximum activity of the enzyme

\section{1,8-cineole}

The starting material, pure 1,8-Cineole, is isolated from Eucalyptus oil, which is obtained from Eucalyptus globulus plants.

*Corresponding author: Andre-Michael Beer, Ruhr University Bochum, Im Vogelsang 5-11, 45527 Hattingen, Germany, Tel: 00492324396456; E-mail: a.beer@klinikum-bochum.de

Received January 31, 2017; Accepted February 16, 2017; Published February 21, 2017

Citation: Beer AM, Zagorchev P, Filipova DM, Lukanov J (2017) Effects of 1,8-Cineole on the Activity of Cyclooxygenase and Cyclooxygenase 1 and Cyclooxygenase 2 Isoforms. Nat Prod Chem Res 5: 253. doi: 10.4172/23296836.1000253

Copyright: $\odot 2017$ Beer AM, et al. This is an open-access article distributed under the terms of the Creative Commons Attribution License, which permits unrestricted use, distribution, and reproduction in any medium, provided the original author and source are credited. 


\section{Statistical methods}

One-way analysis of variance (unifactorial ANOVA) was carried out. Differences were regarded as statistically significant at a level of $\mathrm{p}<0.05$. The statistics programmes Prism 4.0 and Statistic 5.0 were used.

\section{Results}

Figure 1 shows the inhibitory effects of various concentrations of indomethacin ( 0.0001 to $50 \mathrm{mg} / \mathrm{L})$ on the activity of COX-1 and COX-2 isoforms of cyclooxygenase. Both dose-response curves are shown for comparison. The results show that the inhibitory effect of indomethacin is significantly greater on the COX-1 enzyme system than on the COX-2 isoenzyme.

Indomethacin reduced the activity of the cyclooxygenases at concentrations as low as $0.01 \mathrm{mg} / \mathrm{L}$. Cyclooxygenase activity was completely inhibited at a concentration of $6.4 \mathrm{mg} / \mathrm{L}$. The inhibitory effects of 1,8-cineole were apparent at concentrations of $0.05 \mathrm{mg} / \mathrm{L}$ (Figure 1). The maximum inhibitory effect on cyclooxygenase activity was $86 \%$ at an 1,8 -Cineole concentration of about $300 \mathrm{mg} / \mathrm{L}$.

Figure 2 shows the inhibitory effects of 1,8-cineole on the activity of the COX-1 and COX-2 isoforms of cyclooxygenase. The two doseresponse curves show effects similar to that of celecoxib (Figure 3).

Figure 3 shows the inhibitory effect of celecoxib on the activity of COX-1 and COX-2 isoforms of cyclooxygenase. The two dose-response curves show that markedly lower concentrations of celecoxib are required to inhibit the activity of the COX-2 isoform of cyclooxygenase than is the case with the COX-1 isoform.

Table 1 shows the concentrations of 1,8-cineole, indomethacin and celecoxib required to inhibit the activity of the COX-1 isoform by 25 , 50,75 and $100 \%$.

Table 2 shows the concentrations of 1,8-cineole, indomethacin and celecoxib required to inhibit the activity of the COX-2 isoform by 25 , 50,75 and $100 \%$.

Table 3 shows the degree of activity of the COX-1 isoform at the concentrations of the three COX inhibitors that are necessary to inactivate the COX- 2 isoform by $50 \%$.

\section{Discussion}

Undesirable side effects with the clinical use of prostaglandin (PG) synthetase blockers are caused by the fact that all PG synthetase blockers inhibit the activity of both the COX-1 and COX-2 isoforms to

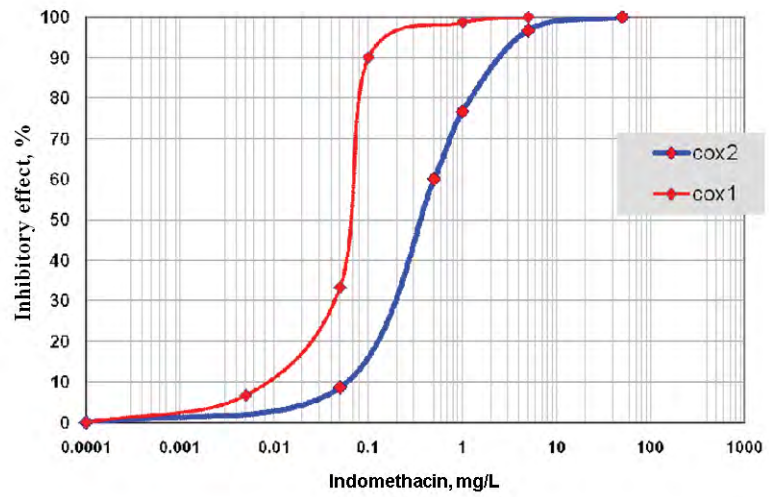

Figure 1: Comparison of the inhibitory effect of various concentrations of indomethacin on the activity of COX-1 and COX-2.

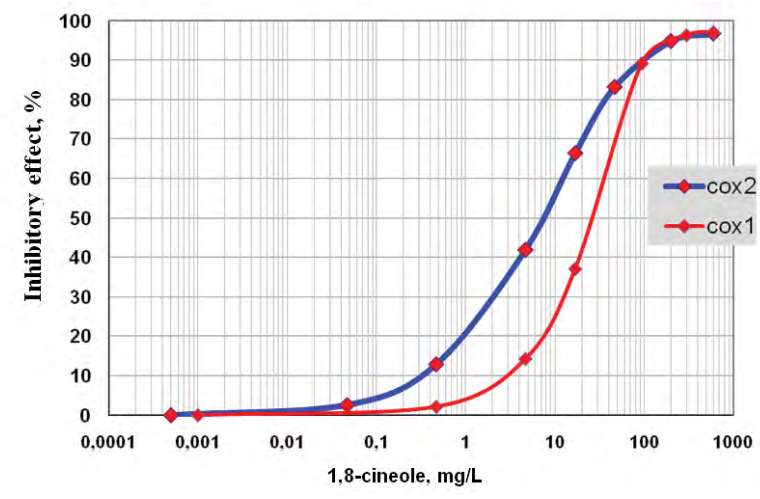

Figure 2: Comparison of the inhibitory effect of various concentrations of 1,8-Cineole on the activity of COX-1 and COX-2.

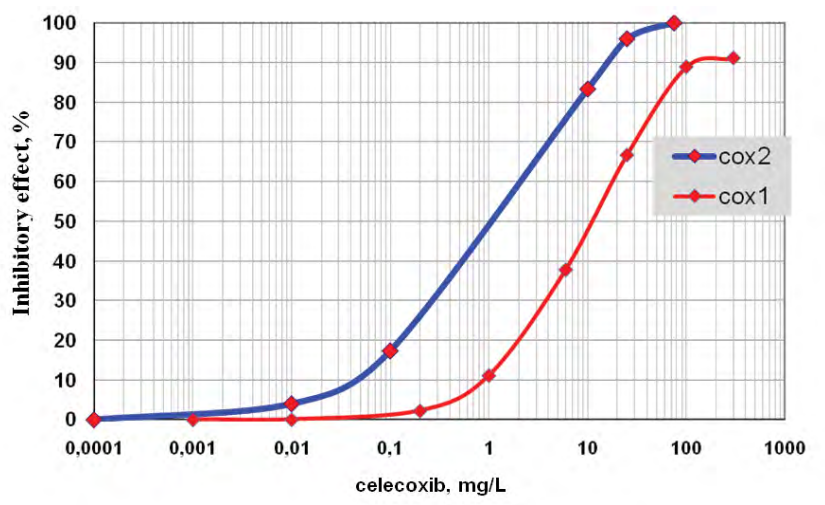

Figure 3: Comparison of the inhibitory effect of various concentrations of celecoxib on the activity of COX-1 and COX-2.

\begin{tabular}{|c|c|c|c|c|}
\hline \multicolumn{5}{|c|}{ Concentrations required to inactivate COX-1 } \\
\hline Substance & $\mathbf{2 5 \%}$ & $\mathbf{5 0} \%$ & $\mathbf{7 5 \%}$ & $\mathbf{1 0 0} \%$ \\
\hline Celecoxib mg/L & $3.2 \pm 0.2$ & $12.3 \pm 0.4$ & $41.5 \pm 0.7$ & - \\
\hline $\mathbf{1 , 8}$-Cineole mg/L & $10.4 \pm 0.2$ & $27.3 \pm 0.3$ & $57.5 \pm 0.7$ & - \\
\hline Indomethacin $\mathbf{~ m g / L ~}$ & $0.03 \pm 0.01$ & $0.06 \pm 0.01$ & $0.08 \pm 0.01$ & $1.10 \pm 0.03$ \\
\hline
\end{tabular}

Table 1: Concentrations of indomethacin, celecoxib and 1,8-Cineole required to achieve $25,50,75$ and $100 \%$ inhibition of COX-1 activity.

\begin{tabular}{|c|c|c|c|c|}
\hline \multicolumn{5}{|c|}{ Concentrations required to inactivate COX-2 } \\
\hline Substance & $\mathbf{2 5 \%}$ & $\mathbf{5 0 \%}$ & $\mathbf{7 5 \%}$ & $\mathbf{1 0 0 \%}$ \\
\hline Celecoxib $\mathbf{~ m g / L ~}$ & $0.18 \pm 0.02$ & $1.2 \pm 0.04$ & $5.7 \pm 0.07$ & $70.4 \pm 0.9$ \\
\hline $\mathbf{1 , 8 - C i n e o l e ~} \mathbf{~ m g / L}$ & $1.4 \pm 0.2$ & $7.8 \pm 0.4$ & $28.7 \pm 0.6$ & - \\
\hline $\begin{array}{c}\text { Indomethacin } \\
\text { mg/L }\end{array}$ & $0.14 \pm 0.01$ & $0.35 \pm 0.01$ & $0.93 \pm 0.01$ & $10.40 \pm 0.03$ \\
\hline
\end{tabular}

Table 2: Concentrations of indomethacin, celecoxib and 1,8-Cineole required to achieve $25,50,75$ and $100 \%$ inhibition of COX-2 activity.

a greater or lesser extent, and thus, selective blockers are not available.

There are two isoforms of cyclooxygenase: COX-1 is an essential enzyme that is present in many body tissues and regulates the production of the prostaglandins in the stomach, which are needed to protect the gastric mucosa. In this context, the release of $\mathrm{PGF}_{2 \mathrm{a}}$ associated with the contractile activity of the gastrointestinal tract is responsible for most of the undesirable side effects.

COX-2 is an enzyme that is expressed predominantly during 
Citation: Beer AM, Zagorchev P, Filipova DM, Lukanov J (2017) Effects of 1,8-Cineole on the Activity of Cyclooxygenase and Cyclooxygenase 1 and Cyclooxygenase 2 Isoforms. Nat Prod Chem Res 5: 253. doi: 10.4172/2329-6836.1000253

\begin{tabular}{|c|c|c|}
\hline Substance & $\begin{array}{c}\text { Concentrations required } \\
\text { for } \mathbf{5 0 \%} \text { inhibition of } \\
\text { COX-2 activity }\end{array}$ & $\begin{array}{c}\text { Level of activity of COX-1 at } \\
\text { the concentrations required } \\
\text { for } \mathbf{5 0 \%} \text { inhibition of COX-2 } \\
\text { activity }\end{array}$ \\
\hline Celecoxib mg/L & $1.20 \pm 0.04$ & Celecoxib $95.4 \%$ \\
\hline $1,8-$-Cineole $\mathrm{mg} / \mathrm{L}$ & $7.80 \pm 0.40$ & 1,8 -Cineole $88.2 \%$ \\
\hline Indomethacin $\mathrm{mg} / \mathrm{L}$ & $0.35 \pm 0.01$ & Indomethacin $3.4 \%$ \\
\hline
\end{tabular}

Table 3: Level of activity of COX-1 at the concentrations required for $50 \%$ inhibition of COX-2 activity.

inflammation, and triggered by cytokines, endotoxins, tumour promoters, growth factors, and tissue injury [10]. The more selectively the COX-2 isoform is blocked, the lower the rate of undesirable side effects.

In order to assess the selectivity of PG synthetase blockers, one can determine the ratio of the molar concentration required for $50 \%$ inactivation of the COX-1 isoform compared with that required for $50 \%$ inactivation of COX-2.

We investigated the effect of 1,8-cineole on the activity of cyclooxygenase and its two isoforms, COX-1 and COX-2. The concentration required to cause a $50 \%$ loss of COX 1 activity is for celecoxib 200 times higher and for 1,8-Cineole 455 times higher than for indomethacin.

In contrast, the concentration required to cause an activity loss of $50 \%$ for COX2 is for celecoxib only three times higher and for 1,8-Cineole only 23 times higher than for indomethacin.

Furthermore, while a $50 \%$ loss of COX 2 activity induced by indomethacin coincides with an almost complete loss of COX1 activity (96.6\% loss of activity), a 50\% loss of activity in COX2 induced by celecoxib or 1,8-Cineole only coincides with a marginal to moderate loss of activity in COX1 (4.5\% or $11,8 \%$ loss of activity). Taken together these results suggest that 1,8 -Cineole preferentially inhibits COX2.

The present results confirm clinical experience obtained from treatment of inflammatory diseases with 1,8-cineole.

\section{Conflicts of Interest}

Prof. J. Lukanov reported receiving financial support from the Klosterfrau Company for reagents and equipment. All other authors declare that they have no conflict of interest.

\section{Acknowledgements}

We thank the Klosterfrau Company for their financial support in making this study possible.

\section{References}

1. Schilcher H, Kammerer S, Wegener T (2007) Leitfaden Phytotherapie. (4th edn), Elsevier, Urban \& Fischer.

2. Forster HB, Niklas H, Lutz S (1980) Antispasmodic effects of some medicinal plants. Planta Med 40: 309-319.

3. Wagner H, Wierer M, Bauer R (1986) In vitro-Hemmung der ProstaglandinBiosynthese durch ätherische Ole und phenolische Verbindungen. Planta Med 52: 184-187.

4. Juergens UR (2014) Anti-inflammatory properties of the monoterpene 1,8-cineole: Current evidence for co-medication in inflammatory airway diseases. Drug Res 64: 638-646.

5. Worth H, Schacher C, Dethlefsen U (2009) Concomitant therapy with Cineole (Eucalyptole) reduces exacerbations in COPD: A placebo-controlled doubleblind trial. Resp Res 10:69.

6. Worth H, Dethlefsen U (2012) Patients with Asthma Benefit from Concomitant Therapy with Cineole: A Placebo-Controlled, Double-Blind Trial. J Asthma 49: 849-853.

7. Juergens UR, Stober M, Schmidt-Schilling L, Kleuver T, Vetter H (1998) Anti-inflammatory effects of eucalyptol (1,8-Cineole) in bronchial asthma: Inhibition of arachidonic acid metabolism in human blood monocytes ex vivo. Eur J Med Res 3: 407-412.

8. Schick V, Schulz HJ (2010) Nichtsteroidale Antirheumatika und Gastrointestinaltrakt. Der Gastroenterologe 5: 461-472.

9. Simmons DL Botting RM, Hla T (2004) Cyclooxygenase isozymes: the biology of prostaglandin synthesis and inhibition. Pharmacol Rev 56: 387-437.

10. Maiden L (2009) Capsule endoscopic diagnosis of nonsteroidal antiinflammatory drug-induced enteropathy. J Gastroenterol 44: 64-71. 